\title{
Brain biomarkers and cognition across adulthood
}

\author{
Angeliki Tsapanou $^{1}$ (이 | Christian Habeck ${ }^{1}$ () | Yunglin Gazes ${ }^{1}$ | \\ Qolamreza Razlighi $^{1}$ ( ) J Jayant Sakhardande ${ }^{1}$ | Yaakov Stern ${ }^{1}$ | Timothy A. Salthouse ${ }^{2}$
}

${ }^{1}$ Cognitive Neuroscience Division, Department of Neurology and the Taub Institute, Columbia University, New York, New York

${ }^{2}$ Department of Psychology, University of

Virginia, Charlottesville, Virginia

\section{Correspondence}

Yaakov Stern, Cognitive Neuroscience Division, Columbia University, 630 W 168th Street, P\&S Box 16, New York, NY 10032.

Email: ys11@cumc.columbia.edu

Funding information

National Institute of Health/National Institute on Aging, Grant/Award Numbers: RF1

AG038465, R01 AG026158

\begin{abstract}
Understanding the associations between brain biomarkers (BMs) and cognition across age is of paramount importance. Five hundred and sixty-two participants (19-80 years old, 16 mean years of education) were studied. Data from structural T1, diffusion tensor imaging, fluid-attenuated inversion recovery, and resting-state functional magnetic resonance imaging scans combined with a neuropsychological evaluation were used. More specifically, the measures of cortical, entorhinal, and parahippocampal thickness, hippocampal and striatal volume, default-mode network and fronto-parietal control network, fractional anisotropy (FA), and white matter hyperintensity (WMH) were assessed. $z$-Scores for three cognitive domains measuring episodic memory, executive function, and speed of processing were computed. Multiple linear regressions and interaction effects between each of the BMs and age on cognition were examined. Adjustments were made for age, sex, education, intracranial volume, and then, further, for general cognition and motion. BMs were significantly associated with cognition. Across the adult lifespan, slow speed was associated with low striatal volume, low FA, and high WMH burden. Poor executive function was associated with low FA, while poor memory was associated with high WMH burden. After adjustments, results were significant for the associations: speed-FA and $\mathrm{WMH}$, memory-entorhinal thickness. There was also a significant interaction between hippocampal volume and age in memory. In age-stratified analyses, the most significant associations for the young group occurred between FA and executive function, $\mathrm{WMH}$, and memory, while for the old group, between entorhinal thickness and speed, and WMH and speed, executive function. Unique sets of BMs can explain variation in specific cognitive domains across adulthood. Such results provide essential information about the neurobiology of aging.
\end{abstract}

\section{KEYWORDS}

aging, brain biomarkers, cognition

\section{1 | INTRODUCTION}

Although relationships between indices of brain and cognition have been examined in aging, the relative relationships of different brain biomarkers (BMs) and cognitive functions across age remain relatively unexplored. Identification of biological factors that are associated with cognition in healthy aging can help our understanding about the underlying mechanisms of aging.
Existing literature on specific BMs has shown an association with cognition, cognitive changes over time and neurodegenerative diseases as well (Jack et al., 2010; Leow et al., 2009). Both BMs and cognition tend to change with age and more dramatically at late stages of adulthood. Significant age-related decreases in total brain volume (BV), as well as in hippocampal and temporal lobe volume occur across the adult age range (Scahill et al., 2003). In healthy aging, white matter volume decreases and is greater than the decrease of the gray matter 
volume (Salat, Kaye, \& Janowsky, 1999). Normal aging has been also associated with declines in specific cognitive domains, mostly in memory, speed of processing, and executive function (Harada, Natelson Love, \& Triebel, 2013). As mentioned in a review (Bressler \& Menon, 2010), the weight of evidence for the association between brain and cognition has now shifted from individual brain areas to the conjoint function of brain areas working together as large-scale networks. Thus, examining more than one individual BM contributes to a more principled and systematic approach to the investigation of cognitive function.

A study by Hedden et al., 2016 in 186 cognitively healthy older adults aged $65-90$ years, revealed that age-related variation in cognition was associated with multiple, preselected BMs, with significant ones varying across cognitive domain. In this study, fractional anisotropy (FA) and striatal volume were strongly associated with processing speed and executive function, whereas hippocampal volume was associated with episodic memory. A different study showed an association between amyloid burden and episodic memory, and white matter hyperintensity $(\mathrm{WMH})$ burden to executive function (Hedden et al., 2012). Studies using magnetic resonance imaging (MRI) and positron emission tomography (PET) scans examining the link between brain measurements and aging across the lifespan revealed significant associations for functional and structural connectivity (Betzel et al., 2014), diffusion tensor imaging (DTI) white matter tract (Lebel et al., 2012; Storsve, Fjell, Yendiki, \& Walhovd, 2016), and subcortical structures (Coupe et al., 2017) with aging. Across the lifespan, a recent examination of subcortical gray matter structures suggested that in a longitudinal aging cohort, the volume of all structures except the caudate and globus pallidus decreased linearly (Narvacan, Treit, Camicioli, Martin, \& Beaulieu, 2017). Thus, differences in brain morphometry are observed with aging, pointing the effect that these might have to cognition. The above studies, both cross-sectional and longitudinal, examined these associations across the whole lifespan using mostly individual measures of the brain trajectories. However, the underlying links between brain modalities and cognition have not yet been fully explored. Furthermore, a lot of interest has been in investigating the role of brain in specific pathologies (DeLisi et al., 1995; Luerding, Weigand, Bogdahn, \& Schmidt-Wilcke, 2008). By taking a step back, and finding the relationships between brain and cognition in neurologically and psychiatric intact adults, we can obtain a more lucid portrait of how brain functions. Examining the relative effect sizes of the BM-cognition associations, as well as the age-related change in these relationships, is of great interest.

Existing literature suggests significant associations between specific BMs and specific aspects of cognition in older adults. However, most of the existing literature has examined a limited amount of BMs, mostly one single biomarker and its association with aging and cognition, and the sample sizes are usually focused on specific age or clinical groups, limiting the generalizability of the results. Examining the associations between multiple BMs and cognition across adulthood in cognitively normal adults can aid the understanding of the mechanisms underlying cognitive function in aging, before a neurodegeneration or a general pathology appears. Thus, we aimed to examine the associations between BMs and cognition, in a large sample of cognitively normal participants, not only in the later stages of adulthood, but across the adult life span. Associations were examined for nine brain measurements. We also examined interaction effects between each of the BMs and age on cognition. These associations were tested in three different cognitive domains.

We hypothesized that BMs such as regional/total BV and thickness, mean FA, functional connectivity, and $\mathrm{WMH}$ burden would be negatively associated with age and positively with cognition. Associations would be significant not only examining the individual BMs but also after evaluating the conjoint role of all the BMs simultaneously in cognition. We expected associations to differ by cognitive domain. Our main goal was to examine the associations between cognitive domains and BMs over and above age-and other demographic factors-although these individual factors could still be age related. In the age-stratified group, we also expected to reveal unique associations between specific cognitive domains and BMs, over and above the relationship to general cognition.

\section{2 | MATERIALS AND METHODS}

The data set consisted of participants from two studies: The reference ability neural network (RANN), and the cognitive reserve (CR) studies. The RANN study was designed to identify networks of brain activity uniquely associated with performance across adulthood of each of the four reference abilities: memory, reasoning, speed of processing, and vocabulary (Habeck et al., 2016). The CR study was designed to elucidate the neural underpinnings of $\mathrm{CR}$ and the concept of brain reserve (Stern, 2012). Both studies share similar recruitment procedure and data collection. All participants were native English speakers, and right-handed. Individuals with any major neurological or psychiatric conditions that could affect their cognitive status were excluded, as were those with mild cognitive impairment or dementia. A score equal or greater than 130 was required on the Mattis Dementia Rating Scale (Mattis, 1988) for the inclusion in the studies, and score no or minimal complaints on a questionnaire about their functionality (Blessed, Tomlinson, \& Roth, 1968). Both RANN and CR have been approved by the Institutional Review Board of Columbia University. More detailed information about the two studies can be found in previous publications (Habeck et al., 2016; Habeck et al., 2017; Razlighi, Habeck, Barulli, \& Stern, 2017; Stern, 2009; Stern, 2012; Stern et al., 2014).

\section{1 | Imaging data}

We used data from structural T1, DTI, fluid-attenuated inversion recovery (FLAIR), and resting-state functional MRI scans. All scans were acquired on the same 3.0 Tesla Philips Achieva MRI scanner. We attempted to use the same BMs analyzed in Hedden et al. as this study is most similar to our approach, analytical method, and aims; however, data for fluoro-deoxy-glucose PET imaging and amyloid burden were not available for our sample. Thus, out of the BMs used based on previous publications and on theoretical importance, 
we incorporated the following nine measurements: cortical, entorhinal, and parahippocampal thickness, hippocampal and striatal volume, FA, default-mode network (DN), fronto-parietal control network (FPCN), and WMH.

\subsection{Volume and cortical thickness}

A T1-weighted Magnetization Prepared Rapid Acquisition Gradient Echo (MPRAGE) scan was acquired with Echo Time/Repetition Time (TE/TR) of $3 / 6.5 \mathrm{~ms}$ and flip angle of $8^{\circ}$, in-plane resolution of $256 \times 256$, field of view of $25.6 \times 25.6 \mathrm{~cm}^{2}$, and $165-180$ slices in axial direction with slice thickness/gap of $1 / 0 \mathrm{~mm}$. FreeSurfer (v5.1.0) software for human brain imaging analysis (http://surfer.nmr.mgh.harvard.edu/) was used for the reconstruction of the T1 scans (Fischl et al., 2002; Fischl et al., 2004). We used the following measures: cortical thickness (total, $\mathrm{mm}$ ), entorhinal thickness (mean right and left, $\mathrm{mm}$ ), parahippocampal thickness (mean right and left, $\mathrm{mm}$ ), hippocampal volume (sum of right and left, $\mathrm{mm}^{3}$ ), and striatal volume (sum of right and left, $\mathrm{mm}^{3}$ ). Thickness in cortical regions was computed using the standard FreeSurfer parcellation (Desikan et al., 2006).

\section{3 | Fractional Anisotropy}

Two sets of DTI images were acquired with each set having 56 directions using these parameters: $b=800 \mathrm{~s} / \mathrm{mm}^{2}$, TE $=69 \mathrm{~ms}$, $\mathrm{TR}=7,645-7,671 \mathrm{~ms}, \quad$ FOV $=22.4 \times 22.4 \mathrm{~cm}^{2}$, flip angle $=90^{\circ}$, in-plane resolution $112 \times 112$ voxels, acquisition time $9 \min 27 \mathrm{~s}$, slice thickness $=2 \mathrm{~mm}$ (no gap), and 75 slices. The two data sets were then concatenated and processed with Tracts Constrained by Underlying Anatomy DTI, distributed as part of the FreeSurfer library that produces 18 major white matter tracts, as described in previous publications (Li, Tsapanou, Qolamreza, \& Gazes, 2018; Yendiki et al., 2011). For each participant, the means of FA for each of the 18 tracts were used to create a single-averaged FA variable, which was entered into subsequent analyses.

\section{4 | Functional connectivity analysis}

A resting-state scan was acquired with TE/TR of 20/2,000 ms and flip angle of $72^{\circ}$, in-plane resolution of $112 \times 112$, field of view of $22.4 \times 22.4 \mathrm{~cm}^{2}$, and 37 slices in axial direction with slice thickness/gap of $3 / 0 \mathrm{~mm}$. After slice-timing correction, realignment, band-pass temporal filtering and scrubbing to correct for motion artifacts, DN, and FPCN intranetwork connectivity were computed as the mean pairwise connectivity in all possible pairs located within the DN and FPCN, as detailed in the taxonomy by Power et al. (2011). We also took the mean framewise displacement from all the functional neuroimaging scans of each participant and used it as a covariance of no interest in all our analyses.

\section{5 | White matter hyperintensity}

FLAIR images for visualization of WMH were used with the following parameters: TR/TI (ms) 11,000/2,800 TE (ms): 125 in plane resolution
$256 \times 189$, FOV $23.0 \times 17.96 \mathrm{~cm}$, and 30 slices with slice thickness/ gap of $4 / 0.5 \mathrm{~mm}$. For the extraction of the $\mathrm{WMH}$, we used the Lesion Segmentation Tool (LST), which is a toolbox for Statistical Parametric Mapping (SPM), able to segment T2 hyperintense lesions in FLAIR images. Lesions were segmented by the lesion growth algorithm (Schmidt et al., 2012) as implemented in the LST version 2.0.15 (www. statistical-modelling.de/lst.html) for SPM. Following standard convention, WMH volumes were log transformed (resulting in a normal distribution) and treated as a continuous variable, with higher values indicating greater $\mathrm{WMH}$ burden.

\section{6 | Neuropsychological factor scores}

Each participant underwent an extensive neuropsychological evaluation. Although some cognitive tests might examine different functions at the same time (Whiteside et al., 2016), for the purposes of the current study, based on previous similar publications (Hedden et al., 2016) and according to our neuropsychological battery, we created three specific cognitive domains measuring; episodic memory, speed of processing, and executive function. The tests used based on our battery were the following:

Episodic memory: Selective Reminding Test, delayed recall, total correct (Buschke \& Fuld, 1974). Speed of processing: The Wechsler Adult Intelligence Scale, Third edition (WAIS-III) Digit-Symbol, total correct (D. 1997), Trail Making Test (TMT)-A, total time (R. 1978). Executive function: fluency: Controlled Word Association (C-F-L) and Category Fluency (animals), total correct (Benton AL 1989). Working memory: WAIS-III LetterNumber Sequencing, total correct (D. 1997). Switching: Incongruent-congruent (mean), TMT B-TMT A (time). Albert Einstein

$z$-scores for each cognitive domain were derived based on the means and standard deviations (SD) of all participants. The $z$-scores were averaged within cognitive domain to derive the composite cognitive score. Higher $z$-score indicates better cognitive performance (reaction times for speed of processing were reversed and $z$-transformed). We also created a general cognition variable by summing up the three above cognitive subcategories. We further ran a principal-axis factor analysis as an alternative indicator of general cognition, and the pattern of the results was similar, thus, we decided to currently report the results of the general cognition-sum variable.

\section{7 | Statistical analysis}

All statistical analyses were performed using the Statistical Package for the Social Sciences (SPSS) 23 (SPSS, Chicago, IL). Nominally significant alpha values were defined as $p<.05$.

We initially explored the correlations between age, each of the cognitive domains and each of the BMs. 
Multiple linear regression models were performed to examine the association between BMs and cognition. All the nine BMs entered simultaneously in the model and were treated as predictors, while the performance on each of the individual cognitive domains, were the outcomes. As this is an exploratory analysis, we chose not to use a strict correction for the multiple comparisons; however, we approached this issue by adding all the measurements simultaneously in the same statistical model. Adjustments were made for age, sex, education, and intracranial volume (ICV). As motion could play a significant role in the association between $\mathrm{BMs}$ and cognition, we further controlled for it. We did not have measured motion for the structural acquisitions; however, for most of the subjects, we had the resting-Blood oxygenation level dependent (BOLD) scan and associated realignment information, which we used as a further covariate.

In order to examine any age-related differences in the relationship between brain morphometry and cognition, we created three age groups (young: 19-39, middle: 40-59, old: 60-80), and performed the same multiple linear regression models with the nine BMs added simultaneously as predictors and each of the cognitive domains as the outcome, stratified by age group. Analysis was first unadjusted and then adjusted for age, sex, education, and ICV. Results are reported for further adjustment by general cognition in order to indicate which of the relations are specific, or unique, to cognitive ability as opposed to being shared with general cognition, and then, motion as well.

We also performed across the whole adult-range generalized linear model analyses to examine interactions between BMs and age for their effect on cognition, with each of the cognitive domains as the dependent variable, and each of the BMs as the predictor. We examined the main effect of all the variables and the interaction effect between each of the BMs and age on the cognitive domains. The models were first unadjusted and then adjusted for the covariates mentioned above.

\section{3 | RESULTS}

Our sample consisted of 562 participants, with $56.8 \%$ women, and had a mean age of 54 (SD: 17) years, and a mean education of 16 (SD: 2.4) years. Demographic, cognitive, and BMs characteristics of the participants, both overall and within the three age ranges, are presented in Table 1.

Table 2 displays the bivariate correlations between age, cognitive domain scores, and brain measures. Age was significantly associated with each of the cognitive domains and each of the BMs as well. The strongest associations were noticed between cortical thickness, striatal volume, and $\mathrm{WMH}$ and all the cognitive domains. Further exploratory relations between age-as a continuous variable-and each of the cognitive domain/each of the BMs was plotted and added in the Supporting Information.

Exploratory multiple linear regression models for the total sample predicting each cognitive domain and using all nine BMs simultaneously showed unique significant associations for the following: speed of processing with striatal volume, FA, and WMH burden; executive function with FA; and episodic memory with $\mathrm{WMH}$ burden (Supporting Information, Table S1). The significance of the whole model was $F: 12.067, p \leq .0001$. After adjusting for the covariates, results remained significant for the associations between speed and both FA and $\mathrm{WMH}$ (Figure 1), while memory was associated with entorhinal thickness (Table 3). The significant associations remained unchanged even after controlling for motion.

TABLE 1 Characteristics of the participants in total and in three age groups

\begin{tabular}{|c|c|c|c|c|}
\hline Age & $\begin{array}{l}19-39, N=147 \\
M: 29(S D: 5)\end{array}$ & $\begin{array}{l}40-59, N=123 \\
M: 51(S D: 5)\end{array}$ & $\begin{array}{l}60-80, N=292 \\
M: 67(S D: 5)\end{array}$ & $\begin{array}{l}\text { Total, } N=562 \\
\text { M:54 (SD:5) }\end{array}$ \\
\hline Sex, women, $N(\%)$ & $98(66.7)$ & $57(46.3)$ & $164(56.2)$ & $319(56.8)$ \\
\hline Education (years), mean (SD) & $15.9(2.3)$ & $16(2.3)$ & $16.3(2.4)$ & $16(2.4)$ \\
\hline Speed, mean $(S D)$ & $0.72(0.7)$ & $0.056(0.8)$ & $-0.36(0.8)$ & $0.0003(0.9)$ \\
\hline Executive, mean (SD) & $0.20(0.6)$ & $-0.012(0.7)$ & $-0.11(0.6)$ & $-0.011(0.6)$ \\
\hline Episodic memory, mean (SD) & $0.62(0.7)$ & $0.13(0.9)$ & $-0.34(1)$ & $0.0(1)$ \\
\hline General cognition, mean (SD) & $1.5(1.4)$ & $0.7(1.7)$ & $-0.8(1.7)$ & $-0.01(1.9)$ \\
\hline Cortical thickness (mm), mean (SD) & $2.63(0.1)$ & $2.56(0.1)$ & $2.48(0.1)$ & $2.54(0.1)$ \\
\hline Entorhinal thickness (mm), mean (SD) & $3.36(0.4)$ & $3.42(0.4)$ & $3.3(0.4)$ & $3.34(0.4)$ \\
\hline Parahippocampal thickness (mm), mean (SD) & $2.81(0.3)$ & $2.75(0.3)$ & $2.7(0.3)$ & $2.7(0.3)$ \\
\hline Hippocampal volume $\left(\mathrm{mm}^{3}\right)$, mean $(S D)$ & $4,220.3(419.7)$ & $4,198.4(448.8)$ & $3,804.2(464.7)$ & $4,013(491.8)$ \\
\hline Striatum volume $\left(\mathrm{mm}^{3}\right)$, mean $(S D)$ & $4,614.8(474.9)$ & $4,279.7(529.2)$ & $3,878.2(562.5)$ & $4,179.9(617.8)$ \\
\hline DN, mean (SD) & $0.090(0.05)$ & $0.070(0.03)$ & $0.064(0.03)$ & $0.073(0.04)$ \\
\hline FPCN, mean (SD) & $0.075(0.05)$ & $0.063(0.04)$ & $0.06(0.04)$ & $0.065(0.04)$ \\
\hline FA, mean (SD) & $0.453(0.02)$ & $0.448(0.02)$ & $0.438(0.02)$ & $0.444(0.02)$ \\
\hline WMH burden, mean (SD) & $0.71(0.9)$ & $1.62(1)$ & $2.67(1)$ & $1.91(1.3)$ \\
\hline
\end{tabular}

Abbreviations: DN, default-mode network; FA, fractional anisotropy; FPCN, fronto-parietal control network; WMH, white matter hyperintensity. 
TAB LE 2 Correlations for age, cognitive domains, and BMs, in the total sample

\begin{tabular}{|c|c|c|c|c|c|c|c|c|c|c|c|c|}
\hline & 1 & 2 & 3 & 4 & 5 & 6 & 7 & 8 & 9 & 10 & 11 & 12 \\
\hline \multicolumn{13}{|l|}{ Age } \\
\hline Speed & $-.54 * *$ & & & & & & & & & & & \\
\hline Episodic memory & $-.43 * *$ & $.36 * *$ & $.31 * *$ & & & & & & & & & \\
\hline Cortical thickness & $-.57 * *$ & $.37 * *$ & $.2 * *$ & $.31 * *$ & & & & & & & & \\
\hline Parahippocampal thickness & $-.21 * *$ & $.22 * *$ & $.16 * *$ & $.21 * *$ & $.35 * *$ & $.27 * *$ & & & & & & \\
\hline Hippocampal volume & $-.41 * *$ & $.28 * *$ & $.21 * *$ & $.18 * *$ & $.33 * *$ & $.3 * *$ & $.27 * *$ & & & & & \\
\hline Striatal volume & $-.55 * *$ & $.38 * *$ & $.24 * *$ & $.23 * *$ & $.36 * *$ & $.18 * *$ & $.10 *$ & $.58 * *$ & & & & \\
\hline 1. DN & $-.29 * *$ & $.12 *$ & .10 & $.14 *$ & $.26 * *$ & .07 & $.13 *$ & .09 & $.16 * *$ & & & \\
\hline FPCN & $-.17 * *$ & .05 & .02 & .10 & $.19 * *$ & .05 & .04 & .03 & $.11 *$ & $.31 * *$ & & \\
\hline
\end{tabular}

Abbreviations: BM, brain biomarker; DN, default-mode network; FA, fractional anisotropy; FPCN, fronto-parietal control network; WMH, white matter hyperintensity.

$*$ Significance level < .05.; **Significance level < .01.

We repeated the multiple linear regression models including all nine BMs simultaneously, stratifying age into three groups: young (19-39), middle (40-59), and old (60-80). Unadjusted results are summarized in Supporting Information, Table S2. Speed of processing was predicted by entorhinal thickness, striatal volume, FA, and WMH in the old group. No significant predictions were noted in the other two age groups. Executive function was associated with entorhinal thickness and FA in the young group, FA in the middle-aged group, and with $\mathrm{WMH}$ in the old group. Episodic memory was associated with hippocampal volume in the young group. No significant predictions were noted in the other two age groups. We then reran these analyses, adjusting for age, gender, education, and ICV (Table 4). Associations remained significant as follows: speed with entorhinal thickness and $\mathrm{WMH}$ in the old group, executive function with FA in the young group, and $\mathrm{WMH}$ in the old group (Figure 2), and episodic memory with hippocampal volume and WMH in the young group (Figure 3). We then added general cognition as an additional covariate (see footnote of Table 4, indicating which associations disappeared upon inclusion of general cognition as covariate). None of the above noted associations between biomarkers and cognition remained significant. However, several new associations were noted. In the young group: executive function with hippocampal volume $(B=0.000, \beta=0.248$, $p=.009)$, and episodic memory with hippocampal volume $(B=0.000$, $\beta=-0.280, p=.006)$. In the old group: episodic memory with $\mathrm{WMH}$ ( $B=0.207, \beta=0.189, p=.028$ ). The significant associations remained unchanged even after controlling for motion. Table 5 sums the significant associations between the BMs and the cognitive domains based on the linear regressions, both across the whole age range and in the three age groups. WMH burden seems to be the better predictor for both the total sample and the young-old groups, followed by FA for the total and the young group, and entorhinal thickness for the total and the old group.
We then performed generalized linear model analyses examining potential interactions between BMs and age in their effect on each of the cognitive domains. These analyses were run across the total sample. Results showed a significant interaction effect between hippocampal volume and age on episodic memory $(B=1.078, p=.031)$ (Figure 4 ), such that as age increased, the association between hippocampal volume and memory performance was stronger. This association remained significant even after adjusting for sex, education, and ICV $(B=9.758, p=.050)$. However, this association did not survive when general cognition and motion were added as further covariates. There were no other significant interaction effects between age and the rest of the BMs on any cognitive domain or general cognition.

\section{4 | DISCUSSION}

Our aim was to examine the associations between BMs and cognition not only in older adults but also extend these analyses to ages 19-80. We found significant associations between specific BMs and cognition in cognitively healthy adults. Our results are generally in agreement with previous studies examining age-related variation in cognition in relation to BMs. In order to sum up the major results of Columbia study and that of Hedden's et al., which is the most close to our study in older adults, we conclude the following: Hedden et al. showed that multiple BMs were associated with age-related variation in cognition in older adults. The largest relationships linked FA, FPCN, and amyloid burden with speed, executive function, and episodic memory. Partial correlation and regression analyses revealed that FA and striatal volume were associated with speed and executive function, while hippocampal volume with episodic memory. Results from the Columbia study showed that in older adults, entorhinal thickness was associated with speed of processing, while WMH was associated with executive function and 


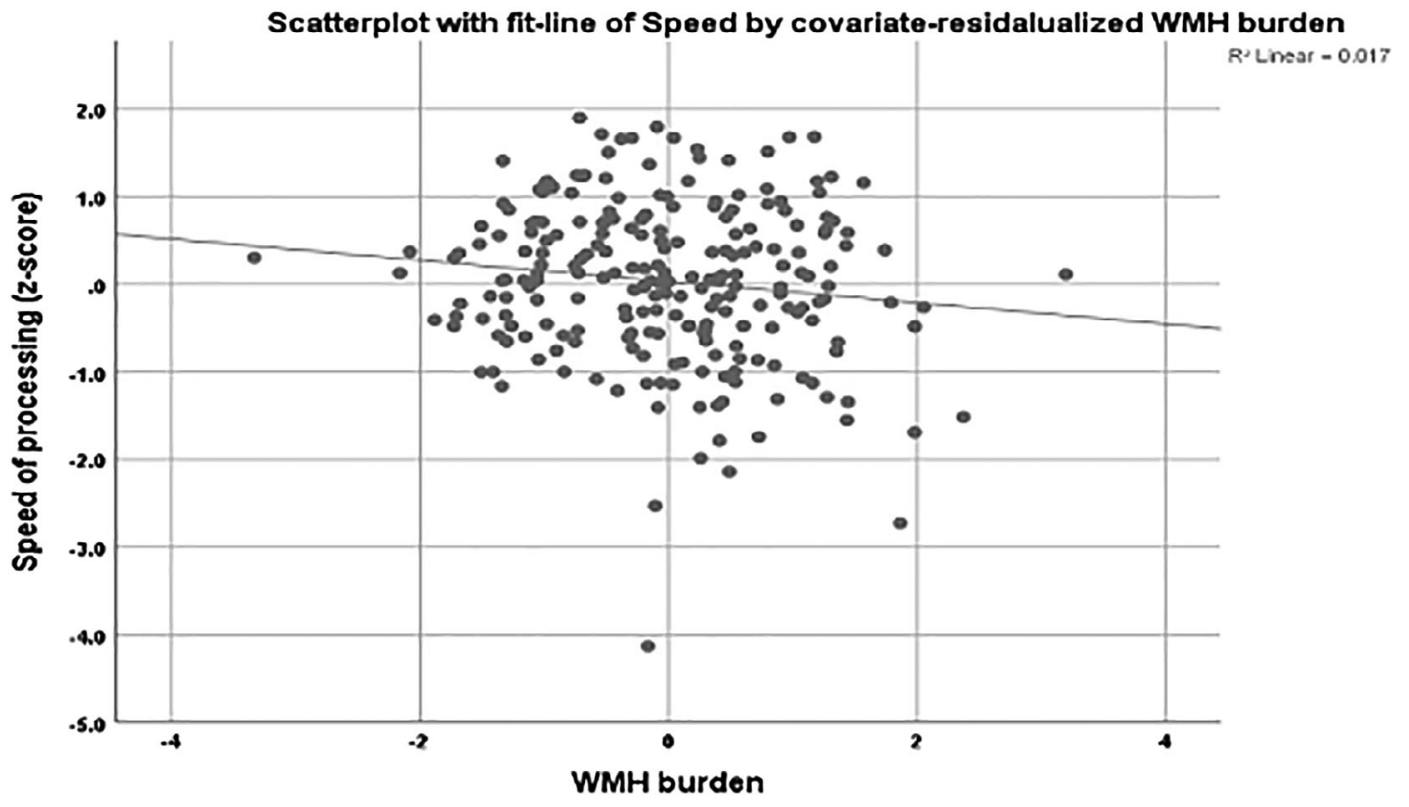

FIGURE 1 Scatterplot for the association between speed of processing and the covariate-residualized WMH burden in the total sample. Age, sex, education, and the remaining BMs were used as covariates. Lower speed of processing was associated with higher WMH burden in the total sample. BM, brain biomarker; WMH, white matter hyperintensity

TABLE 3 Multiple linear regressions for the association between BMs and each of the cognitive domains, for the total sample, adjusted for age, sex, education, and ICV

\begin{tabular}{|c|c|c|c|c|c|c|c|c|c|}
\hline \multirow{3}{*}{$\begin{array}{l}\text { BMs } \\
\text { Age }\end{array}$} & \multicolumn{3}{|c|}{ Speed B, $\boldsymbol{\beta}, \boldsymbol{p}$} & \multicolumn{3}{|c|}{ Executive $B, \beta, p$} & \multicolumn{3}{|c|}{ Episodic memory B, $\beta, p$} \\
\hline & \multicolumn{3}{|c|}{$R^{2}=.391$} & \multicolumn{3}{|c|}{$R^{2}=.158$} & \multicolumn{3}{|c|}{$R^{2}=.216$} \\
\hline & -.017 & -.322 & .002 & -.003 & -.086 & .469 & -.017 & -.300 & .011 \\
\hline Education & .030 & .078 & .169 & .040 & .155 & .019 & .015 & .037 & .572 \\
\hline Sex & .253 & .140 & .044 & .080 & .066 & .409 & -.008 & -.004 & .957 \\
\hline ICV & 8.684 & .151 & .063 & 8.680 & .227 & .017 & -2.979 & -.005 & .957 \\
\hline Entorhinal thickness & .222 & .094 & .119 & .150 & .096 & .175 & .352 & .144 & .038 \\
\hline Parahippocampal thickness & .012 & .004 & .949 & .067 & .030 & .657 & .235 & .067 & .308 \\
\hline Hippocampal volume & .000 & -.068 & .381 & -1.722 & -.014 & .875 & .000 & -.084 & .339 \\
\hline Striatal volume & .000 & .110 & .215 & -4.889 & -.049 & .633 & -2.411 & -.016 & .878 \\
\hline WMH burden & -.133 & -.182 & .023 & -.041 & -.084 & .356 & -.130 & -.170 & .067 \\
\hline
\end{tabular}

Abbreviations: BM, brain biomarker; DN, default-mode network; FA, fractional anisotropy; FPCN, fronto-parietal control network; ICV, intracranial volume; $\mathrm{WMH}$, white matter hyperintensity.

memory. Expanding across the adulthood, speed of processing was associated with striatal volume, FA, and $\mathrm{WMH}$, executive function with $\mathrm{FA}$, and memory with WMH.

Different cognitive functions have been linked to either very specific brain areas or a combination of different brain modalities, with new atlases being developed recently mapping the association between brain and cognition (Varoquaux et al., 2018). FA and WMH were significantly associated with cognitive performance in our analyses. These two BMs have been associated with cognition in previous research. It was suggested that FA can be a useful measure for early detection of age-related cognitive change, and specifically for changes in executive function (Grieve, Williams, Paul, Clark, \& Gordon, 2007). Another study showed that areas with decreased FA in deep-WMH predicted poor general cognition, as opposed to highgrade periventricular hyperintensities, suggesting the involvement of cortico-subcortical circuits in cognitive deficits only in deep-WMH 
TAB LE 4 Multiple linear regression for the association between BMs and each of the cognitive domains, stratified by age group, adjusted for age, sex, education, ICV, and then additionally for general cognition $\left({ }^{\mathrm{a}}\right)$

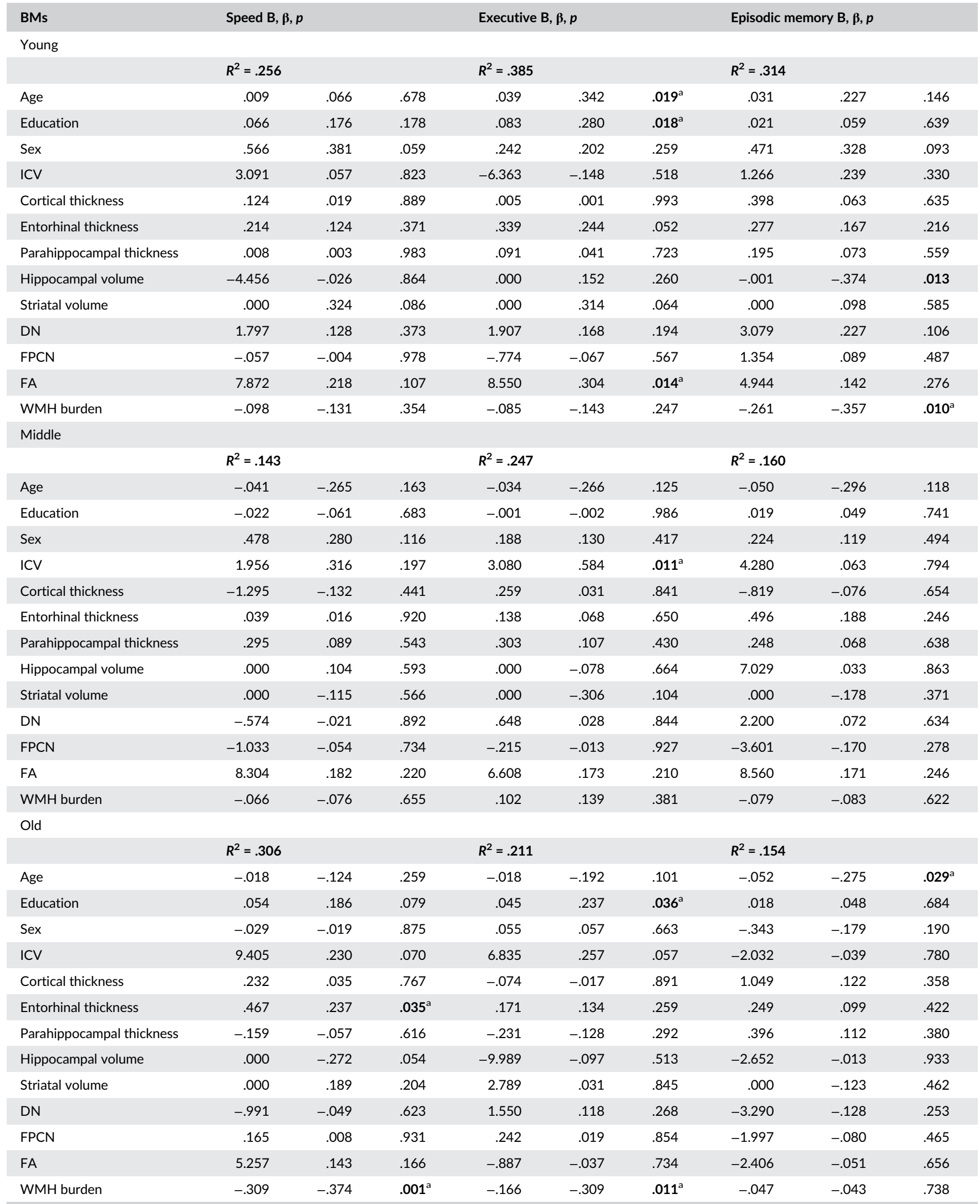

Abbreviations: BM, brain biomarker; DN, default-mode network; FA, fractional anisotropy; FPCN, fronto-parietal control network; WMH, white matter hyperintensity.

${ }^{\text {aT }}$ These associations did not survive after controlling also for general cognition. 


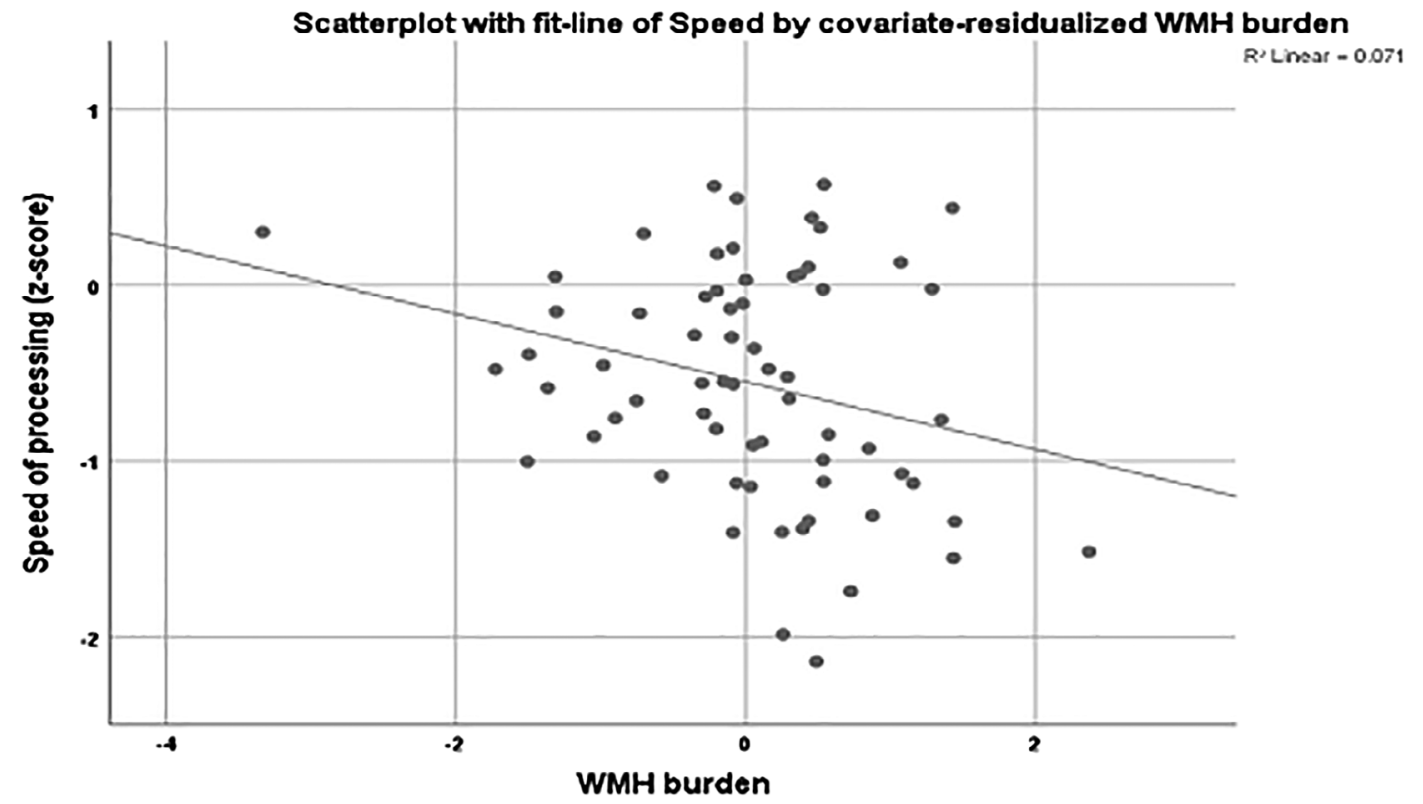

FIGURE 2 Scatterplot for the association between speed of processing and the covariate-residualized WMH burden in the old group. Age, sex, education, and the remaining BMs were used as covariates. Lower speed of processing was associated with higher WMH burden in the old group. BM, brain biomarker; $\mathrm{WMH}$, white matter hyperintensity

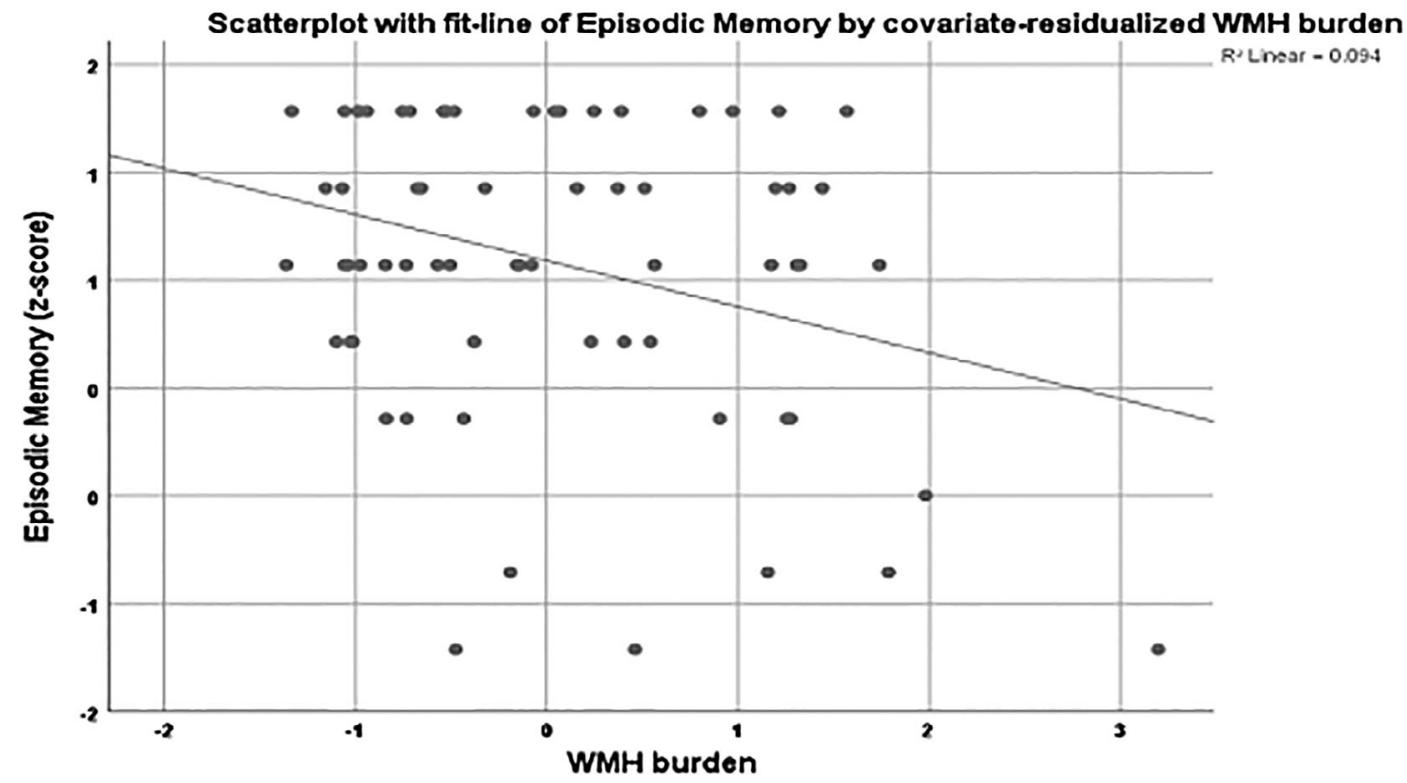

FIGURE 3 Scatterplot for the association between episodic memory and the covariate-residualized WMH burden in the young group. Age, sex, education, and the remaining BMs were used as covariates. Lower performance in episodic memory was associated with higher WMH burden in the young group. BM, brain biomarker; $\mathrm{WMH}$, white matter hyperintensity

(Soriano-Raya et al., 2014). Our results suggest a significant association of $\mathrm{FA}$ and $\mathrm{WMH}$ burden with different cognitive domains across the whole adult age range, in cognitively healthy participants. Furthermore, our results reveal the significance of FA and WMH for cognition over and above other BMs, in contrast to most of the existing studies that focused on individual BMs only. Specifically, in our study, low FA was associated with low speed of processing, while high $\mathrm{WMH}$ burden was associated with slow speed of processing and low episodic memory across age. As both diffusion imaging data and signal abnormalities in the white matter are not regionally localized, it is reasonable that these BMs are associated with performance in multiple cognitive domains.

Our results indicate that the association between hippocampal volume and memory becomes stronger as people age. Subtle differences in the hippocampal size could be more influential in older adults, especially in the cognitive domain of memory. Moreover, we could hypothesize that within the context of lower hippocampal volume, 
TAB LE 5 *Significant associations between the BMs and the cognitive domains, based on the linear regression models, in the total sample, and in the three age groups. Model adjusted for age, sex, education, and ICV

\begin{tabular}{|c|c|c|c|c|c|c|c|c|c|c|c|c|}
\hline & \multicolumn{4}{|c|}{ Speed } & \multicolumn{4}{|c|}{ Executive } & \multicolumn{4}{|c|}{ Episodic memory } \\
\hline \multicolumn{13}{|l|}{ Cortical thickness } \\
\hline Entorhinal thickness & & & & $*$ & & & & & $*$ & & & \\
\hline \multicolumn{13}{|c|}{ Parahippocampal thickness } \\
\hline Hippocampal volume & & & & & & & & & & * & & \\
\hline \multicolumn{13}{|l|}{ DN } \\
\hline \multicolumn{13}{|l|}{ FPCN } \\
\hline FA & $*$ & & & & & * & & & & & & \\
\hline WMH burden & * & & & $*$ & & & & $*$ & & $*$ & & \\
\hline
\end{tabular}

Abbreviations: BM, brain biomarker; DN, default-mode network; FA, fractional anisotropy; FPCN, fronto-parietal control network; WMH, white matter hyperintensity.

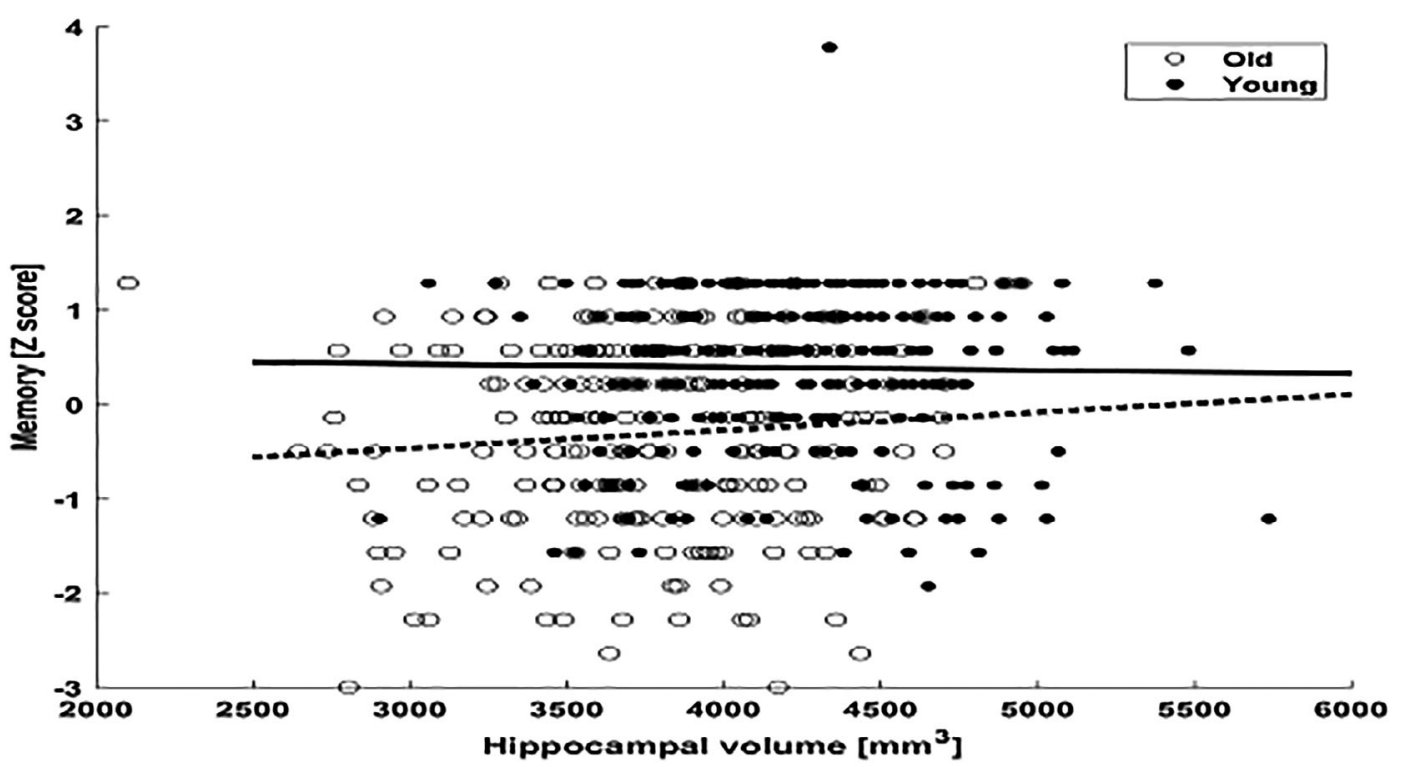

FIGURE 4 Scatterplot for the interaction effect between hippocampal volume and age on episodic memory, in total sample with a median split age of 60 years old

people with even slightly higher volume perform better in memory. An extensive review (Van Petten, 2004) reports similarly interesting results of the relationship between memory and hippocampal volume, with the association appearing quite weak for older adults. Based on this review, results seem to vary across age, while statistical methodology appears to play a significant role to this. We cannot also overlook the fact that our domain of episodic memory is consisted of a single score. Creating a memory domain consisted of a combination of more memory scores could probably be more sensitive to the measurement of the specific cognitive function.

Greater variance in executive function $\left(R^{2}=.385\right)$ and episodic memory $\left(R^{2}=.314\right)$ can be better explained by the specific set of BMs in young adults than in older ones $\left(R^{2}=.211, R^{2}=.154\right.$, respectively) in our study. Results for speed of processing, however, revealed that greater variance in the specific domain can be explained by the specific BMs in older adults $\left(R^{2}=.306\right)$ than the younger group $\left(R^{2}=.256\right)$. Associations remained significant even after adjusting for age, sex, education, and ICV. Other factors not included in the model might play a significant role for the remaining variance in cognition. For instance, biomarkers like amyloid burden (McConathy \& Sheline, 2015) or cerebral blood flow as measured by arterial spin labeling (De Vis et al., 2018) have been associated with cognition and could explain behavioral performance in different cognitive domains. We used a priori chosen markers, mostly associated with cognition in older adults; however, future studies including a greater variety of $\mathrm{BMs}$, the role, and interaction of which in cognition has not been fully explored yet, will provide us with important information about the core of the neurobiology of aging. Overall, we found that with age 
some associations between specific BMs and cognitive domains are quite strong, while others not. Both cognition and brain morphometry change over time, with some cognitive domains like speed of processing having a slower decline or even getting improved-like vocabulary-(Kemper \& Kemtes, 2000), and some brain modalities having similarly increased decline-mostly in the limbic system(Gunbey et al., 2014) or not a significant change over time. Our results are in accordance with existing literature suggesting that age-related changes in cognition vary considerably across individuals and cognitive domains, with some cognitive functions appearing more susceptible than others to the effects of aging (Glisky, 2007).

We repeated our multiple regression analyses with adjusting for a global cognition covariate, in order to evaluate whether the BMs' relationships to a specific cognitive domain exceed what is already provided by global cognition. A few studies have explored this shared and unique variance in cognition, and based on Salthouse et al., only by simultaneous consideration of both shared and unique influences is it possible to accurately identify the contribution of unique influences (Salthouse et al., 2015). In the age-stratified analyses, after adjusting for general cognition, the observed associations between specific BMs and cognitive domains were no longer significant, although the association was significant for general cognition. However, in some cases, new associations with specific domains appeared. Thus, it might be more accurate to give more emphasis to the significant associations between each of the cognitive domains and the BMs after controlling for general cognition. These analyses showed that for young adults, executive function and episodic memory were associated with hippocampal volume. For older adults, episodic memory was associated with WMH burden. We could thus note that the general cognitive status might affect the unique contribution of each individual domain. Hence, individual cognitive domains, although examining different cognitive performances, they might all be connected to each other.

There are some limitations in the current study. The crosssectional design does not provide information about the direction of causality of the results. Furthermore, the a priori selection of BMs might exclude some other biomarkers associated with cognitive performance and which could explain greater age-related variation in cognition. We implemented only previously studied and broadly available analysis techniques, as replication and standardization across large-scale studies is important for establishing robust relationships between brain morphometry and cognitive performance.

There are also some significant strengths. Most of the existing studies are limited in their ecological validity by examining specific subgroups. By expanding to the whole age range of adulthood, we can early define brain measures that are associated with cognition, and probably, contribute to a healthy aging. Thus, strength of the current study is that we examined the association between BMs and cognition not only in the elderly but also across the whole adult age range. Furthermore, compared to previous publications, we used a larger sample size. Another strength of the study is that we performed multiple regressions taking into account the role of the general cognition, providing us with more accurate information about the unique contribution of the BMs. Finally, by using multiple linear regression models, we adjusted for all the BMs, and we made further adjustments for other factors that may contribute to the association between brain and cognition (age, sex, education, and ICV).

In conclusion, specific BMs are associated with cognitive performance, in cognitively normal adults across the whole adult age range. WMH seems to be the strongest predictor of cognition, as it is linked to all the three cognitive domains and most associations remain significant both across the whole age range and separately in the different age groups. Results could have significant implications in public health and provide important information about the factors need to be controlled in order to ensure well-maintained brain function.

\section{ACKNOWLEDGMENTS}

This work was supported by the National Institute of Health/National Institute of Aging (NIH/NIA) (grant numbers R01 AG026158 and RF1 AG038465).

\section{ORCID}

Angeliki Tsapanou (D) https://orcid.org/0000-0003-0471-5786

Christian Habeck (D) https://orcid.org/0000-0001-9961-7446

Qolamreza Razlighi (D) https://orcid.org/0000-0001-9588-8062

\section{REFERENCES}

Benton, A. L., \& Hamsher, K. (1989). Multilingual aphasia examination. lowa City, IA: AJA Associates.

Betzel, R. F., Byrge, L., He, Y., Goni, J., Zuo, X. N., \& Sporns, O. (2014). Changes in structural and functional connectivity among resting-state networks across the human lifespan. Neurolmage, 102(Pt. 2), 345-357.

Blessed, G., Tomlinson, B. E., \& Roth, M. (1968). The association between quantitative measures of dementia and of senile change in the cerebral grey matter of elderly subjects. The British Journal of Psychiatry, 114, 797-811.

Bressler, S. L., \& Menon, V. (2010). Large-scale brain networks in cognition: Emerging methods and principles. Trends in Cognitive Sciences, 14, 277-290.

Buschke, H., \& Fuld, P. A. (1974). Evaluating storage, retention, and retrieval in disordered memory and learning. Neurology, 24, 1019-1025.

Coupe, P., Catheline, G., Lanuza, E., Manjon, J. V., \& Initiative Alzheimer's Disease Neuroimaging. (2017). Towards a unified analysis of brain maturation and aging across the entire lifespan: A MRI analysis. Human Brain Mapping, 38, 5501-5518.

De Vis, J. B., Peng, S. L., Chen, X., Li, Y., Liu, P., Sur, S., ... Lu, H. (2018). Arterial-spin-labeling (ASL) perfusion MRI predicts cognitive function in elderly individuals: A 4-year longitudinal study. Journal of Magnetic Resonance Imaging, 48(2), 449-458. https://doi.org/10.1002/jmri.25938.

DeLisi, L. E., Tew, W., Xie, S., Hoff, A. L., Sakuma, M., Kushner, M., ... Grimson, R. (1995). A prospective follow-up study of brain morphology and cognition in first-episode schizophrenic patients: Preliminary findings. Biological Psychiatry, 38, 349-360.

Desikan, R. S., Segonne, F., Fischl, B., Quinn, B. T., Dickerson, B. C., Blacker, D., ... Killiany, R. J. (2006). An automated labeling system for subdividing the human cerebral cortex on MRI scans into gyral based regions of interest. Neurolmage, 31, 968-980.

Fischl, B., Salat, D. H., Busa, E., Albert, M., Dieterich, M., Haselgrove, C., ... Dale, A. M. (2002). Whole brain segmentation: Automated labeling of neuroanatomical structures in the human brain. Neuron, 33, 341-355. 
Fischl, B., Salat, D. H., van der Kouwe, A. J., Makris, N., Segonne, F., Quinn, B. T., \& Dale, A. M. (2004). Sequence-independent segmentation of magnetic resonance images. Neurolmage, 23(Suppl. 1), S69-S84.

Glisky, E. L. (2007). Changes in cognitive function in human aging. In D. R. Riddle (Ed.), Brain aging: Models, methods, and mechanisms. Boca Raton, FL: CRC Press Chapter 1.

Grieve, S. M., Williams, L. M., Paul, R. H., Clark, C. R., \& Gordon, E. (2007). Cognitive aging, executive function, and fractional anisotropy: A diffusion tensor MR imaging study. AJNR. American Journal of Neuroradiology, 28, 226-235.

Gunbey, H. P., Ercan, K., Findikoglu, A. S., Bulut, H. T., Karaoglanoglu, M., \& Arslan, H. (2014). The limbic degradation of aging brain: A quantitative analysis with diffusion tensor imaging. The Scientific World Journal, 2014 196513.

Habeck, C., Gazes, Y., Razlighi, Q., Steffener, J., Brickman, A., Barulli, D., ... Stern, Y. (2016). The reference ability neural network study: Life-time stability of reference-ability neural networks derived from task maps of young adults. Neurolmage, 125, 693-704.

Habeck, C., Razlighi, Q., Gazes, Y., Barulli, D., Steffener, J., \& Stern, Y. (2017). Cognitive reserve and brain maintenance: Orthogonal concepts in theory and practice. Cerebral Cortex, 27, 3962-3969.

Harada, C. N., Natelson Love, M. C., \& Triebel, K. L. (2013). Normal cognitive aging. Clinics in Geriatric Medicine, 29, 737-752.

Hedden, T., Mormino, E. C., Amariglio, R. E., Younger, A. P., Schultz, A. P., Becker, J. A., ... Rentz, D. M. (2012). Cognitive profile of amyloid burden and white matter hyperintensities in cognitively normal older adults. The Journal of Neuroscience, 32, 16233-16242.

Hedden, T., Schultz, A. P., Rieckmann, A., Mormino, E. C., Johnson, K. A., Sperling, R. A., \& Buckner, R. L. (2016). Multiple brain markers are linked to age-related variation in cognition. Cerebral Cortex, 26, 1388-1400.

Jack, C. R., Jr., Knopman, D. S., Jagust, W. J., Shaw, L. M., Aisen, P. S., Weiner, M. W., ... Trojanowski, J. Q. (2010). Hypothetical model of dynamic biomarkers of the Alzheimer's pathological cascade. Lancet Neurology, 9, 119-128.

Kemper, S., \& Kemtes, K. (2000). Aging and message production and comprehension. In D. Park \& N. Schwarz (Eds.), Cognitive aging: A primer (Vol. 2000, p. 197). Philadelphia, PA: Psychology Press.

Lebel, C., Gee, M., Camicioli, R., Wieler, M., Martin, W., \& Beaulieu, C. (2012). Diffusion tensor imaging of white matter tract evolution over the lifespan. Neurolmage, 60, 340-352.

Leow, A. D., Yanovsky, I., Parikshak, N., Hua, X., Lee, S., Toga, A. W., ... Initiative Alzheimer's Disease Neuroimaging. (2009). Alzheimer's disease neuroimaging initiative: A one-year follow up study using tensor-based morphometry correlating degenerative rates, biomarkers and cognition. Neurolmage, 45, 645-655.

Li, P., Tsapanou, A., Qolamreza, R. R., \& Gazes, Y. (2018). White matter integrity mediates decline in age-related inhibitory control. Behavioura Brain Research, 339, 249-254.

Luerding, R., Weigand, T., Bogdahn, U., \& Schmidt-Wilcke, T. (2008). Working memory performance is correlated with local brain morphology in the medial frontal and anterior cingulate cortex in fibromyalgia patients: Structural correlates of pain-cognition interaction. Brain, 131, 3222-3231.

Mattis, S. (1988). Dementia rating scale (DRS). Odessa, FL: Psychological Assessment Resources.

McConathy, J., \& Sheline, Y. I. (2015). Imaging biomarkers associated with cognitive decline: A review. Biological Psychiatry, 77, 685-692.

Narvacan, K., Treit, S., Camicioli, R., Martin, W., \& Beaulieu, C. (2017). Evolution of deep gray matter volume across the human lifespan. Human Brain Mapping, 38, 3771-3790.

Power, J. D., Cohen, A. L., Nelson, S. M., Wig, G. S., Barnes, K. A., Church, J. A., ... Petersen, S. E. (2011). Functional network organization of the human brain. Neuron, 72, 665-678.
Razlighi, Q. R., Habeck, C., Barulli, D., \& Stern, Y. (2017). Cognitive neuroscience neuroimaging repository for the adult lifespan. Neurolmage, 144, 294-298.

Reitan, R. (1978). Manual for administration of Neuropsychological test batteries for adults and children. San Antonio, TX: Psychological Corporation.

Salat, D. H., Kaye, J. A., \& Janowsky, J. S. (1999). Prefrontal gray and white matter volumes in healthy aging and Alzheimer disease. Archives of Neurology, 56, 338-344.

Salthouse, T. A., Habeck, C., Razlighi, Q., Barulli, D., Gazes, Y., \& Stern, Y. (2015). Breadth and age-dependency of relations between cortical thickness and cognition. Neurobiology of Aging, 36, 3020-3028.

Scahill, R. I., Frost, C., Jenkins, R., Whitwell, J. L., Rossor, M. N., \& Fox, N. C. (2003). A longitudinal study of brain volume changes in normal aging using serial registered magnetic resonance imaging. Archives of Neurology, 60, 989-994.

Schmidt, P., Gaser, C., Arsic, M., Buck, D., Forschler, A., Berthele, A., ... Muhlau, M. (2012). An automated tool for detection of FLAIRhyperintense white-matter lesions in multiple sclerosis. Neurolmage, 59, 3774-3783.

Soriano-Raya, J. J., Miralbell, J., Lopez-Cancio, E., Bargallo, N., Arenillas, J. F., Barrios, M., ... Mataro, M. (2014). Tract-specific fractional anisotropy predicts cognitive outcome in a community sample of middle-aged participants with white matter lesions. Journal of Cerebral Blood Flow and Metabolism, 34, 861-869.

Stern, Y. (2009). Cognitive reserve. Neuropsychologia, 47, 2015-2028.

Stern, Y. (2012). Cognitive reserve in ageing and Alzheimer's disease. Lancet Neurology, 11, 1006-1012.

Stern, Y., Habeck, C., Steffener, J., Barulli, D., Gazes, Y., Razlighi, Q., ... Salthouse, T. (2014). The reference ability neural network study: Motivation, design, and initial feasibility analyses. Neurolmage, 103, 139-151.

Storsve, A. B., Fjell, A. M., Yendiki, A., \& Walhovd, K. B. (2016). Longitudinal changes in white matter tract integrity across the adult lifespan and its relation to cortical thinning. PLoS One, 11, e0156770.

Van Petten, C. (2004). Relationship between hippocampal volume and memory ability in healthy individuals across the lifespan: Review and meta-analysis. Neuropsychologia, 42, 1394-1413.

Varoquaux, G., Schwartz, Y., Poldrack, R. A., Gauthier, B., Bzdok, D., Poline, J. B., \& Thirion, B. (2018). Atlases of cognition with large-scale human brain mapping. PLoS Computational Biology, 14, e1006565.

Wechsler, D. (1997). Wechsler Adult Intelligence Scale (3rd ed., pp. 684-690). San Antonio, TX: Harcourt Assessment.

Whiteside, D. M., Kealey, T., Semla, M., Luu, H., Rice, L., Basso, M. R., \& Roper, B. (2016). Verbal fluency: Language or executive function measure? Applied Neuropsychology. Adult, 23, 29-34.

Yendiki, A., Panneck, P., Srinivasan, P., Stevens, A., Zollei, L., Augustinack, J., ... Fischl, B. (2011). Automated probabilistic reconstruction of whitematter pathways in health and disease using an atlas of the underlying anatomy. Frontiers in Neuroinformatics, 5, 23.

\section{SUPPORTING INFORMATION}

Additional supporting information may be found online in the Supporting Information section at the end of this article.

How to cite this article: Tsapanou A, Habeck C, Gazes Y, et al. Brain biomarkers and cognition across adulthood. Hum Brain Mapp. 2019;40:3832-3842. https://doi.org/10.1002/ hbm. 24634 